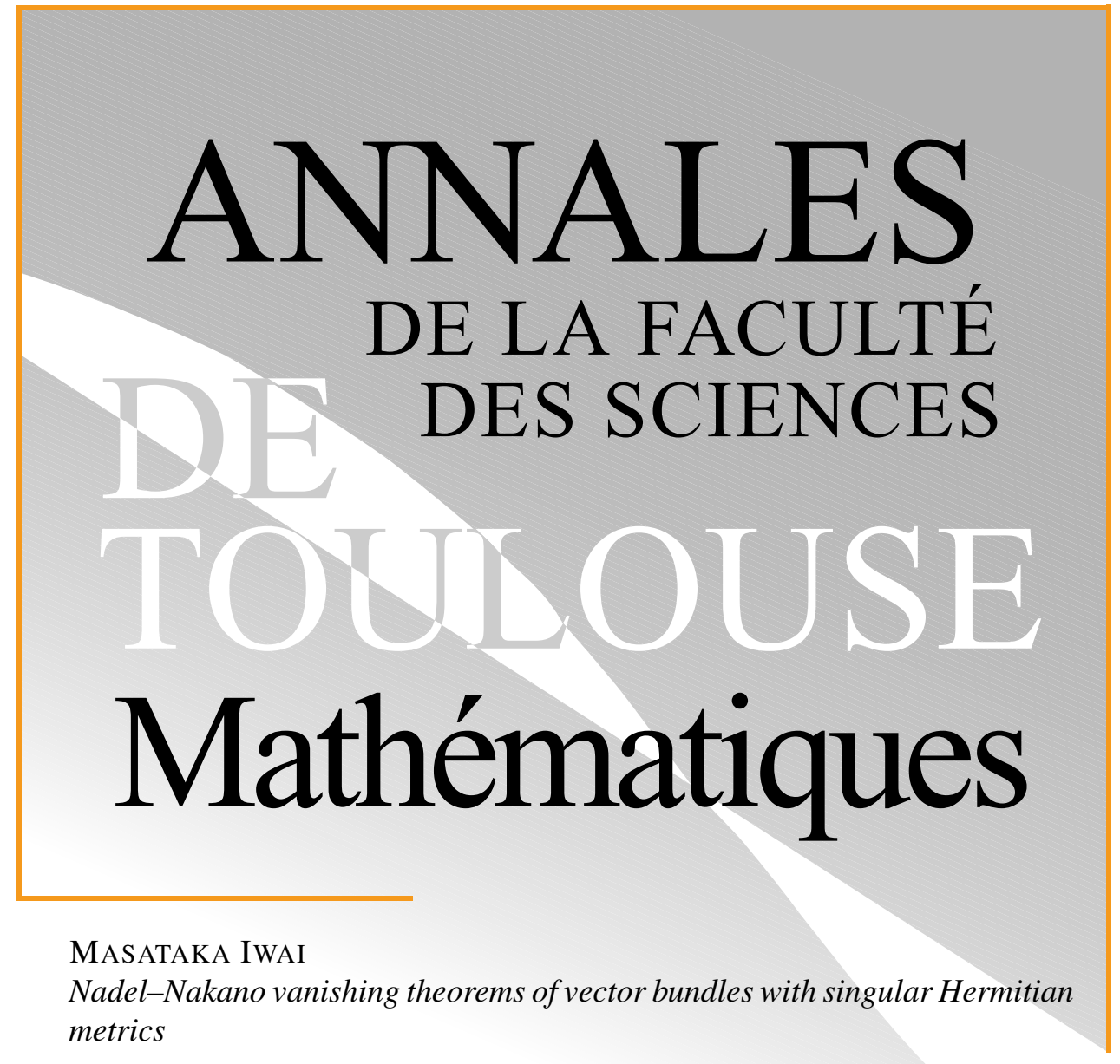

Tome XXX, no 1 (2021), p. 63-81.

https://doi.org/10.5802/afst.1666

(C) Université Paul Sabatier, Toulouse, 2021.

L'accès aux articles de la revue «Annales de la faculté des sciences de Toulouse Mathématiques » (http://afst.centre-mersenne.org/) implique l'accord avec les conditions générales d'utilisation (http://afst.centre-mersenne.org/legal/). Les articles sont publiés sous la license CC-BY 4.0.

(c)

Publication membre du centre

Mersenne pour l'édition scientifique ouverte MERSENNE http://www.centre-mersenne.org/ 


\title{
Nadel-Nakano vanishing theorems of vector bundles with singular Hermitian metrics ${ }^{(*)}$
}

\author{
MASATAKA IWAI ${ }^{(1)}$
}

\begin{abstract}
We study a singular Hermitian metric of a vector bundle. First, we prove that the sheaf of locally square integrable holomorphic sections of a vector bundle with a singular Hermitian metric, which is a higher rank analog of a multiplier ideal sheaf, is coherent under some assumptions. Second, we prove a Nadel-Nakano type vanishing theorem of a vector bundle with a singular Hermitian metric. We do not use an approximation technique of a singular Hermitian metric. We apply these theorems to a singular Hermitian metric induced by holomorphic sections and a big vector bundle, and we obtain a generalization of Griffiths' vanishing theorem. Finally, we show a generalization of Ohsawa's vanishing theorem.
\end{abstract}

RÉsumÉ. - Nous étudions une métrique hermitienne singulière d'un fibré vectoriel. Premièrement, nous montrons que le faisceau de sections holomorphes localement carrées et holomorphes d'un faisceau vectoriel avec une métrique hermitienne singulière, qui est un analogue de rang supérieur d'un faisceau d'idéaux multiplicateurs, est cohérent sous certaines hypothèses. Deuxièmement, nous prouvons un théorème d'annulation de type Nadel-Nakano d'un faisceau de vecteurs avec une métrique hermitienne singulière Nous n'utilisons pas une technique d'approximation d'une métrique hermitienne singulière. Nous appliquons ces théorèmes à une métrique hermitienne singulière induite par des sections holomorphes et un fibré vectoriel gros, et nous obtenons une généralisation du théorème d'annulation de Griffiths. Enfin, nous montrons une généralisation du théorème d'annulation d'Ohsawa.

\section{Introduction}

The aim of this paper is to study the vanishing theorem of a vector bundle with a singular Hermitian metric. Here is a brief history of a singular

(*) Reçu le 19 juin 2018, accepté le 27 mai 2019.

(1) Graduate School of Mathematical Sciences, The University of Tokyo, 3-8-1

Komaba, Tokyo, 153-8914, Japan — masataka@ms.u-tokyo.ac.jp

This work was supported by the Program for Leading Graduate Schools, MEXT, Japan. Support was also provided in the form of a JSPS KAKENHI Grant (\#17J04457).

Article proposé par Vincent Guedj. 
Hermitian metric of a vector bundle. A singular Hermitian metric of a vector bundle is a higher rank analog of a singular Hermitian metric of a line bundle. The singular Hermitian metric was originated by de Cataldo [5], and was later defined in a different way by Berndtsson and Păun [3]. We adopt the definition of a singular Hermitian metric of a vector bundle in [3]. They also defined the notion of a singular Hermitian metric with positive curvature, called positively curved. In [19], Păun and Takayama proved that a direct image sheaf of an $m$-th relative canonical line bundle $f_{*}\left(m K_{X / Y}\right)$ can be endowed with a positively curved singular Hermitian metric for any fibration $f: X \rightarrow Y$. Recently Cao and Păun [4] used this result to prove Iitaka's conjecture when the base space is an Abelian variety. For more details, we refer the reader to [18].

Although a singular Hermitian metric of a vector bundle has been investigated in many papers (for example $[3,14,15,19,20]$ ), there exist few results on vanishing theorems for vector bundles with singular Hermitian metrics. We explain the details of the investigations of a singular Hermitian metric of a vector bundle below. Let $(X, \omega)$ be a compact Kähler manifold and $(E, h)$ be a vector bundle with a singular Hermitian metric. In [5], the sheaf of locally square integrable holomorphic sections of $E$ with respect to $h$, denoted by $E(h)$, is defined as

$$
E(h)_{x}=\left\{f_{x} \in \mathcal{O}(E)_{x}:\left|f_{x}\right|_{h}^{2} \in L_{l o c}^{1}\right\} x \in X,
$$

which is a higher rank analog of a multiplier ideal sheaf. In this paper, we will denote by $\mathcal{O}(E)_{x}$ the stalk of $E$ at $x$, defined by $\lim _{x \in U} H^{0}(U, E)$. We consider the following problems.

PROBLEM 1.1.

(1) Is $E(h)$ a coherent sheaf?

(2) Does there exist a Nadel-Nakano type vanishing theorem, that is, the vanishing of the cohomology group $H^{q}\left(X, K_{X} \otimes E(h)\right)$ for any $q \geqslant 1$ if $h$ has some positivity?

We do not know if $E(h)$, unlike a multiplier ideal sheaf, is coherent, In [5], de Cataldo proved that $E(h)$ is coherent and a Nadel-Nakano type vanishing theorem if $h$ has an approximate sequence of smooth Hermitian metrics $\left\{h_{\mu}\right\}$ satisfying $h_{\mu} \uparrow h$ pointwise and $\sqrt{-1} \Theta_{E, h_{\mu}}-\eta \omega \otimes I d_{E} \geqslant 0$ in the sense of Nakano for some positive and continuous function $\eta$. However, $h$ does not always have such an approximate sequence (see [15, Example 4.4]). Therefore these problems are open.

Nonetheless, we can provide a partial answer to Problem 1.1. First we prove the coherence of $E(h)$ under some assumptions. 
THEOREM 1.2. - Let $(X, \omega)$ be a Kähler manifold and $(E, h)$ be a holomorphic vector bundle on $X$ with a singular Hermitian metric. We assume the following conditions.

(1) There exists a proper analytic subset $Z$ such that his smooth on $X \backslash Z$.

(2) $h e^{-\zeta}$ is a positively curved singular Hermitian metric on E for some continuous function $\zeta$ on $X$.

(3) There exists a real number $C$ such that $\sqrt{-1} \Theta_{E, h}-C \omega \otimes \operatorname{Id}_{E} \geqslant 0$ on $X \backslash Z$ in the sense of Nakano.

Then the sheaf $E(h)$ is coherent.

Next we study the cohomology group $H^{q}\left(X, K_{X} \otimes E(h)\right)$ for any $q \geqslant 1$. We prove a vanishing theorem and an injectivity theorem for vector bundles with singular Hermitian metrics under some assumptions.

ThEOREM 1.3. - Let $(X, \omega)$ be a compact Kähler manifold and $(E, h)$ be a holomorphic vector bundle on $X$ with a singular Hermitian metric. We assume the following conditions.

(1) There exists a proper analytic subset $Z$ such that his smooth on $X \backslash Z$.

(2) $h e^{-\zeta}$ is a positively curved singular Hermitian metric on E for some continuous function $\zeta$ on $X$.

(3) There exists a positive number $\epsilon>0$ such that $\sqrt{-1} \Theta_{E, h}-\epsilon \omega \otimes$ $\operatorname{Id}_{E} \geqslant 0$ on $X \backslash Z$ in the sense of Nakano.

Then $H^{q}\left(X, K_{X} \otimes E(h)\right)=0$ holds for any $q \geqslant 1$.

TheOREm 1.4. - Let $(X, \omega)$ be a compact Kähler manifold, $(E, h)$ be a holomorphic vector bundle on $X$ with a singular Hermitian metric and $\left(L, h_{L}\right)$ be a holomorphic line bundle with a smooth metric. We assume the following conditions.

(1) There exists a proper analytic subset $Z$ such that his smooth on $X \backslash Z$.

(2) $h e^{-\zeta}$ is a positively curved singular Hermitian metric on $E$ for some continuous function $\zeta$ on $X$.

(3) $\sqrt{-1} \Theta_{E, h} \geqslant 0$ on $X \backslash Z$ in the sense of Nakano.

(4) There exists a positive number $\epsilon>0$ such that $\sqrt{-1} \Theta_{E, h}-$ $\epsilon \sqrt{-1} \Theta_{L, h_{L}} \otimes \operatorname{Id}_{E} \geqslant 0$ on $X \backslash Z$ in the sense of Nakano.

Let $s$ be a non zero section of $L$. Then for any $q \geqslant 0$, the multiplication homomorphism

$$
\times s: H^{q}\left(X, K_{X} \otimes E(h)\right) \longrightarrow H^{q}\left(X, K_{X} \otimes L \otimes E(h)\right)
$$

is injective. 
Therefore we proved a Nadel-Nakano type vanishing theorem with some assumptions. If $E$ is a holomorphic line bundle, these theorems were proved in [9]. We point out we do not use an approximation sequence of a singular Hermitian metric to show these theorems.

Some applications are indicated as follows. First, we treat a singular Hermitian metric induced by holomorphic sections, as proposed by Hosono [15, Chapter 4]. By calculating the curvature of this metric, we prove that we can apply Theorem 1.3 to Hosono's example. Therefore we can apply a Nadel-Nakano type vanishing theorem even if $h$ does not have an approximate sequence such as [5]. Second, we generalize Griffiths' vanishing theorem. That is, $H^{q}\left(X, K_{X} \otimes \operatorname{Sym}^{m}(E) \otimes \operatorname{det} E\right)=0$ holds for any $m \geqslant 0$ and $q \geqslant 1$ if $E$ is an ample vector bundle. We treat the case when $E$ is a big vector bundle. If $E$ is a big vector bundle with some assumptions, $\operatorname{Sym}^{m}(E) \otimes \operatorname{det} E$ can be endowed with a singular Hermitian metric $h_{m}$ satisfying assumptions such as those in Theorem 1.3 (see Section 5.2). Therefore $H^{q}\left(X, K_{X} \otimes\left(\operatorname{Sym}^{m}(E) \otimes \operatorname{det} E\right)\left(h_{m}\right)\right)=0$ holds for any $m \geqslant 0$ and $q \geqslant 1$.

Finally, we generalize Ohsawa's vanishing theorem.

THEOREM 1.5. - Let $(X, \omega)$ be a compact Kähler manifold and $(E, h)$ be a holomorphic vector bundle on $X$ with a singular Hermitian metric. Let $\pi: X \rightarrow W$ be a proper surjective holomorphic map to an analytic space with a Kähler form $\sigma$. We assume the following conditions.

(1) There exists a proper analytic subset $Z$ such that his smooth on $X \backslash Z$.

(2) $h e^{-\zeta}$ is a positively curved singular Hermitian metric on E for some continuous function $\zeta$ on $X$.

(3) $\sqrt{-1} \Theta_{E, h}-\pi^{*} \sigma \otimes \operatorname{Id}_{E} \geqslant 0$ on $X \backslash Z$ in the sense of Nakano.

Then $H^{q}\left(W, \pi_{*}\left(K_{X} \otimes E(h)\right)\right)=0$ holds for any $q \geqslant 1$.

If $h$ is smooth, this theorem was proved by Ohsawa [17].

The organization of this paper is as follows. In Section 2, we review some of the standard facts on vector bundles, singular Hermitian metrics and $L^{2}$ estimates. In Section 3, we prove Theorem 1.2. The proof is based on [8, Lemma 5] and [5, Proposition 4.1.3]. Since $h$ has singularities along $Z$, we apply the $L^{2}$ estimate only outside $Z$. In Section 4 , we prove Theorem 1.3 and 1.4. Based on [9, Claim 1], we prove the cohomology isomorphism between the cohomology group of $K_{X} \otimes E(h)$ on $X$ and the $L^{2}$ cohomology group on $X \backslash Z$ by using Čech cohomology. From this isomorphism, it is easy to prove Theorem 1.3 and 1.4. In Section 5, we treat some applications. For example, we treat a singular Hermitian metric induced by holomorphic 
sections and prove a generalization of Griffiths' vanishing theorem. In Section 6 , we prove a generalization of Ohsawa's vanishing theorem by using the methods of [17, Theorem 3.1] and Section 4.

\section{Acknowledgments}

The author would like to thank his supervisor Prof. Shigeharu Takayama for helpful comments and enormous support. He would like to thank Genki Hosono and Takahiro Inayama for useful comments about the applications in Section 5 .

\section{Preliminaries}

\subsection{Hermitian metrics on vector bundles}

We briefly explain definitions and notations of smooth Hermitian metrics of vector bundles.

We will denote by $(X, \omega)$ a compact Kähler manifold and denote by $E$ a holomorphic vector bundle of rank $r$ on $X$. For any point $x \in X$, we take a system of local coordinates $\left(V ; z_{1}, \ldots, z_{n}\right)$ near $x$. Let $h$ be a smooth metric on $E$ and let $e_{1}, \ldots, e_{r}$ be a local orthogonal frame of $E$ near $x$. We denote by

$$
\sqrt{-1} \Theta_{E, h}=\sqrt{-1} \sum_{1 \leqslant j, k \leqslant n, 1 \leqslant \lambda, \mu \leqslant r} c_{j k \lambda \mu} \mathrm{d} z_{j} \wedge \mathrm{d} \bar{z}_{k} \otimes e_{\lambda}^{*} \otimes e_{\mu}
$$

the Chern curvature tensor. For any $u=\sum_{1 \leqslant j \leqslant n, 1 \leqslant \lambda \leqslant r} u_{j \lambda} \frac{\partial}{\partial z_{j}} \otimes e_{\lambda} \in T_{x} X \otimes$ $E_{x}$, we denote by

$$
\theta_{E, h}(u)=\sum_{1 \leqslant j, k \leqslant n, 1 \leqslant \lambda, \mu \leqslant r} c_{j k \lambda \mu} u_{j \lambda} \bar{u}_{k \mu}
$$

and

$$
\theta_{\omega \otimes \operatorname{Id}_{E}}(u)=\sum_{1 \leqslant j, k \leqslant n, 1 \leqslant \lambda \leqslant r} \omega_{j k} u_{j \lambda} \bar{u}_{k \lambda},
$$

where $\omega=\sqrt{-1} \sum_{1 \leqslant j, k \leqslant n} \omega_{j k} \mathrm{~d} z_{j} \wedge \mathrm{d} \bar{z}_{k}$.

Definition 2.1 ([6, Chapter $7 \S 6])$. — For any real number $C$, we write $\sqrt{-1} \Theta_{E, h} \geqslant C \omega \otimes \operatorname{Id}_{E}$ in the sense of Nakano if $\theta_{E, h}(u)-C \theta_{\omega \otimes \operatorname{Id}_{E}}(u) \geqslant 0$ for any $u \in T X \otimes E$. 
Next, we review the definitions of singular Hermitian metrics. For more details, we refer the reader to [18, Section 2]. Let $H_{r}$ be the set of $r \times r$ semipositive definite Hermitian matrices and $\bar{H}_{r}$ be the space of semipositive, possibly unbounded Hermitian forms on $\mathbb{C}^{r}$.

Definition 2.2 ([18, Definition 2.8 and Definition 2.9]).

(1) A singular Hermitian metric $h$ on $E$ is defined to be a locally measurable map with values in $\bar{H}_{r}$ such that $0<\operatorname{det} h<+\infty$ almost everywhere.

(2) A singular Hermitian metric $h$ on $E$ is said to be negatively curved if the function $\log |v|_{h}^{2}$ is plurisubharmonic for any local section $v$ of $E$.

(3) A singular Hermitian metric $h$ on $E$ is said to be positively curved if the dual singular Hermitian metric $h^{*}={ }^{t} h^{-1}$ on the dual vector bundle $E^{*}$ is negatively curved.

We prove the following lemma of a positively curved singular Hermitian metric.

LemMA 2.3. - For any point $x \in X$, we take a system of local coordinate $\left(V ; z_{1}, \ldots, z_{n}\right)$ near $x$ and take a local holomorphic frame $e_{1}, \ldots, e_{r}$ of $E$ on $V$. Let $U \Subset V$ be an open set near $x$. We assume there exists a continuous function $\zeta$ on $X$ such that he $e^{-\zeta}$ is a positively curved singular Hermitian metric on $E$. Then there exists a positive number $M_{U}$ such that for any $u \in H^{0}(V, E)$

$$
|u|_{h}^{2} \geqslant M_{U} \sum_{1 \leqslant i \leqslant r}\left|u_{i}\right|^{2}
$$

holds on $U$, where $u=\sum_{1 \leqslant i \leqslant r} u_{i} e_{i}$.

Proof. - We may assume $u=u_{1} e_{1}$. By [14, Chapter 16], we obtain

$$
|u|_{h e^{-\zeta}}(z)=\sup _{f \in E_{z}^{*}} \frac{|f(u)|(z)}{|f|_{\left(h e^{-\zeta}\right)^{*}}} \geqslant \frac{\left|e_{1}^{*}(u)\right|(z)}{\left|e_{1}^{*}\right|_{\left(h e^{-\zeta}\right)^{*}}}=\frac{\left|u_{1}\right|(z)}{\left|e_{1}^{*}\right|_{\left(h e^{-\zeta}\right)^{*}}}
$$

for any $z \in V$. Since $h e^{-\zeta}$ is positively curved, $\left|e_{1}^{*}\right|_{\left(h e^{-\zeta}\right)^{*}}$ is a plurisubharmonic function on $V$. Therefore $\left|e_{1}^{*}\right|_{\left(h e^{-\zeta}\right) *}$ is bounded above on $U$. We take a positive number $M_{1}$ such that $\left|e_{1}^{*}\right|_{\left(h e^{-\zeta}\right)^{*}} \leqslant M_{1}$, then we have $|u|_{h e^{-\zeta}} \geqslant \frac{\left|u_{1}\right|}{M_{1}}$. Since $e^{\zeta}$ is a positive continuous function, we can take a positive number $M$ such that $e^{\zeta} \geqslant M$ on $X$. We set $M_{U}:=\frac{M^{2}}{M_{1}^{2}}$ and we obtain

$$
|u|_{h}^{2}=|u|_{h e^{-\zeta}}^{2} e^{2 \zeta} \geqslant M_{U}\left|u_{1}\right|^{2},
$$

which completes the proof. 


\section{2. $L^{2}$ estimates and harmonic integrals on complete Kähler man- ifolds}

We need an $L^{2}$ estimate on a complete Kähler manifold. Let $Y$ be a complete Kähler manifold, $\omega^{\prime}$ be a (not necessarily complete) Kähler form and $(E, h)$ be a vector bundle with a smooth Hermitian metric. The $L^{2}$ space $L_{n, q}^{2}(Y, E)_{\omega^{\prime}, h}$ is defined by the set of $E$-valued $(n, q)$ forms with measurable coefficients on $Y$ such that $\int_{Y}|f|_{\omega^{\prime}, h}^{2} \mathrm{~d} V_{\omega^{\prime}}<+\infty$, where $\mathrm{d} V_{\omega^{\prime}}:=\omega^{\prime n} / n$ ! is a volume form on $Y$.

Theorem 2.4 ([6, Chapter $7 \S 7$ and Chapter 8 \$6] [7, Lemme 3.2 and Théorème 4.1]). - Under the conditions stated above, we also assume that there exists a positive number $\epsilon>0$ such that $\sqrt{-1} \Theta_{E, h} \geqslant \epsilon \omega^{\prime} \otimes \operatorname{Id}_{E}$ in the sense of Nakano. Then for any $q \geqslant 1$ and any $g \in L_{n, q}^{2}(Y, E)_{\omega^{\prime}, h}$ such that $\bar{\partial} g=0$, there exists $f \in L_{n, q-1}^{2}(Y, E)_{\omega^{\prime}, h}$ such that $\bar{\partial} f=g$ and

$$
\int_{Y}|f|_{\omega^{\prime}, h}^{2} \mathrm{~d} V_{\omega^{\prime}} \leqslant \frac{1}{q \epsilon} \int_{Y}|g|_{\omega^{\prime}, h}^{2} \mathrm{~d} V_{\omega^{\prime}}
$$

We use a fact of harmonic integrals to prove Theorem 1.4. For more details, we refer the reader to [9, Section 2] or [6, Chapter 8]. The maximal closed extension of the $\bar{\partial}$ operator determines a densely defined closed operator $\bar{\partial}: L_{n, q}^{2}(Y, E)_{\omega^{\prime}, h} \rightarrow L_{n, q+1}^{2}(Y, E)_{\omega^{\prime}, h}$. Then we obtain the following orthogonal decomposition.

Theorem 2.5 ([9, Section 3], [6, Chapter 8]).

$$
L_{n, q}^{2}(Y, E)_{\omega^{\prime}, h}=\overline{\operatorname{Im} \bar{\partial}} \oplus \mathcal{H}^{n, q}(Y, E) \oplus \overline{\operatorname{Im} \bar{\partial}_{\omega^{\prime}, h}^{*}}
$$

holds, where $\bar{\partial}_{\omega^{\prime}, h}^{*}$ is the Hilbert space adjoint of $\bar{\partial}$ and $\mathcal{H}^{n, q}(Y, E)$ is the set of harmonic forms defined by

$$
\mathcal{H}^{n, q}(Y, E):=\left\{f \in L_{n, q}^{2}(Y, E)_{\omega^{\prime}, h}: \bar{\partial} f=\bar{\partial}_{\omega^{\prime}, h}^{*} f=0\right\} .
$$

\section{Coherence of $E(h)$}

We prove Theorem 1.2.

Proof. - We may assume that $X$ is a unit ball in $\mathbb{C}^{n}, E=X \times \mathbb{C}^{r}$, and $\omega$ is a standard Euclidean metric. Let $e_{1}, \ldots, e_{r}$ be a local holomorphic frame of $E$ on $X$. We take an open ball $U \subset \subset X$. It is enough to show that there exists a coherent sheaf $\mathcal{F}$ on $U$ such that $E(h)_{x}=\mathcal{F}_{x}$ for any $x \in U$.

We will denote by $\mathcal{G}$ the space of holomorphic sections $g \in H^{0}(U, E)$ such that $\int_{U}|g|_{h}^{2} \mathrm{~d} V_{\omega}<\infty$. We consider the evaluation map $\pi:\left.\mathcal{G} \otimes_{\mathbf{C}} \mathcal{O}_{U} \rightarrow E\right|_{U}$. 
We define $\mathcal{F}:=\operatorname{Im}(\pi)$. By Noether's Lemma (see [12, Chapter $5 \S 6]$ ), $\mathcal{F}$ is a coherent sheaf on $U$.

Claim 3.1. - For any $x \in U$ and any positive integer $k$,

$$
\mathcal{F}_{x}+E(h)_{x} \cap m_{x}^{k} \cdot E_{(x)}=E(h)_{x}
$$

holds, where $m_{x}$ is a maximal ideal of $\mathcal{O}_{x}$.

We postpone the proof of Claim 3.1 and conclude the proof of Theorem 1.2. We fix $x \in U$. By the Artin-Rees lemma, there exists a positive integer $l$ such that

$$
E(h)_{x} \cap m_{x}^{k} \cdot E_{(x)}=m_{x}^{k-l}\left(E(h)_{x} \cap m_{x}^{l} \cdot E_{(x)}\right)
$$

holds for any $k>l$. Therefore by Claim 3.1, we have

$$
E(h)_{x}=\mathcal{F}_{x}+E(h)_{x} \cap m_{x}^{k} \cdot E_{(x)} \subset \mathcal{F}_{x}+m_{x} \cdot E(h)_{x} \subset E(h)_{x} .
$$

By Nakayama's lemma, we obtain $E(h)_{x}=\mathcal{F}_{x}$, which completes the proof.

We now prove Claim 3.1.

Proof. - It is easy to check that $\mathcal{F}_{x}+E(h)_{x} \cap m_{x}^{k} \cdot E_{(x)} \subset E(h)_{x}$; therefore, we show that $E(h)_{x} \subset \mathcal{F}_{x}+E(h)_{x} \cap m_{x}^{k} \cdot E_{(x)}$.

We take $f=\sum_{i} f_{i} e_{i} \in E(h)_{x}$. Then there exists an open neighborhood $W \subset \subset U$ near $x$ such that $f_{i}$ is a holomorphic function on $W$ and $\int_{W}|f|_{h}^{2} \mathrm{~d} V_{\omega}<+\infty$. Let $\rho$ be a cut-off function on $W$. We note that $\bar{\partial}(\rho f)$ is an $E$-valued $(0,1)$ smooth form such that $\int_{X}|\rho f|_{\omega, h}^{2} \mathrm{~d} V_{\omega}<+\infty$. We define the plurisubharmonic function $\varphi_{k}$ to be $\varphi_{k}(z)=(n+k) \log |z-x|^{2}+C|z|^{2}$ such that

$\sqrt{-1} \Theta_{E, h}+\sqrt{-1} \partial \bar{\partial} \varphi_{k} \otimes \operatorname{Id}_{E} \geqslant \omega \otimes \operatorname{Id}_{E}$ on $X \backslash Z$ in the sense of Nakano, where $C$ is some positive constant. Since $\rho$ is a cut-off function, we obtain $\int_{X}|\bar{\partial}(\rho f)|_{\omega, h}^{2} e^{-\varphi_{k}} \mathrm{~d} V_{\omega}<+\infty$.

Since $X \backslash Z$ is complete by [7, Théorème 0.2], there exists an $E$-valued $(0,0)$ form $F=\sum_{i} F_{i} e_{i}$ on $X \backslash Z$ such that

$$
\int_{X \backslash Z}|F|_{h}^{2} e^{-\varphi_{k}} \mathrm{~d} V_{\omega} \leqslant \int_{X}|\bar{\partial}(\rho f)|_{\omega, h}^{2} e^{-\varphi_{k}} \mathrm{~d} V_{\omega}<+\infty \quad \text { and } \quad \bar{\partial} F=\bar{\partial}(\rho f)
$$

by Theorem 2.4. Here we may regard $\bar{\partial}(\rho f)$ as an $(n, 1)$ form $\bar{\partial}(\rho f) \mathrm{d} z^{1} \wedge$ $\cdots \wedge \mathrm{d} z^{n}$ on $X$ with values in $-K_{X}$.

Let $G:=\rho f-F=\sum_{i} G_{i} e_{i}$, which is an $E$-valued $(0,0)$ form on $X \backslash Z$. We obtain

$$
\int_{X \backslash Z}|G|_{h}^{2} \mathrm{~d} V_{\omega}<+\infty \quad \text { and } \quad \bar{\partial} G=0 .
$$


By Lemma 2.3 we have $\sum_{i} \int_{U \backslash Z}\left|G_{i}\right|^{2} \mathrm{~d} V_{\omega}<+\infty$, and therefore $G_{i}$ extends to the whole of $U$ and $G_{i}$ is holomorphic on $U$ by the Riemann extension theorem. Hence we obtain $G \in \mathcal{G}$ and $G_{x} \in \mathcal{F}_{x}$.

Let $W^{\prime}$ be the set of interior points in $\{z \in U: \rho(z)=1\}$; then we have $F=f-G$ on $W^{\prime} \backslash Z$. Then $F$ extends on $W^{\prime}$ and $F$ is holomorphic on $W^{\prime}$. It is obvious that $F_{x} \in E(h)_{x}$ from $f_{x} \in E(h)$ and $G_{x} \in \mathcal{F}_{x} \subset E(h)_{x}$. By $\int_{X \backslash Z}|F|_{h}^{2} e^{-\varphi_{k}} \mathrm{~d} V_{\omega}<+\infty$ and Lemma 2.3, we have

$$
\sum_{i} \int_{W^{\prime}}\left|F_{i}\right|^{2} e^{-(n+k) \log |z-x|^{2}} \mathrm{~d} V_{\omega}<+\infty .
$$

Therefore we obtain $\left(F_{i}\right)_{x} \in m_{x}^{k}$ and $F_{x} \in m_{x}^{k} \cdot E_{(x)}$.

Thus we have $f_{x}=G_{x}+F_{x} \in \mathcal{F}_{x}+E(h)_{x} \cap m_{x}^{k} \cdot E_{(x)}$, which completes the proof of Claim 3.1.

\section{Vanishing theorems and injectivity theorems}

Let $(X, \omega)$ be a compact Kähler manifold and $(E, h)$ be a holomorphic vector bundle with a singular Hermitian metric on $X$. We assume the conditions (1)-(3) in Theorem 1.2. We will denote $Y:=X \backslash Z$. By [9, Section 3], there exists a complete Kähler form $\omega^{\prime}$ on $Y$ such that $\omega^{\prime} \geqslant \omega$ on $Y$. We study the cohomology group $H^{q}\left(X, K_{X} \otimes E(h)\right)$.

THEOREM 4.1. - Under the conditions stated above, we obtain the following isomorphism:

$$
H^{q}\left(X, K_{X} \otimes E(h)\right) \cong \frac{L_{n, q}^{2}(Y, E)_{\omega^{\prime}, h} \cap \operatorname{Ker} \bar{\partial}}{\operatorname{Im} \bar{\partial}}
$$

for any $q \geqslant 0$.

Proof. - The proof will be divided into three steps.

Step 1: Setup. - Let $\mathcal{U}=\left\{U_{j}\right\}_{j \in \Lambda}$ be a finite Stein cover of $X$. By Theorem 1.2, the sheaf cohomology $H^{q}\left(X, K_{X} \otimes E(h)\right)$ is isomorphic to the Čech cohomology $H^{q}\left(\mathcal{U}, K_{X} \otimes E(h)\right)$. If necessary we take $U_{j}$ small enough, we may assume that there exists a Stein open set $V_{j}$, a smooth plurisubharmonic function $\varphi_{j}$ on $V_{j}$ and a positive number $C_{j}>0$ such that

(1) $U_{j} \subset \subset V_{j}$

(2) $C_{j}^{-1}<e^{-\varphi_{j}}<C_{j}$ on $V_{j}$, and

(3) $\sqrt{-1} \Theta_{E, h}+\sqrt{-1} \partial \bar{\partial} \varphi_{j} \geqslant \omega^{\prime} \otimes \operatorname{Id}_{E}$ on $V_{j} \backslash Z$ 
for any $j \in \Lambda$. We set $U_{i_{0} i_{1} \ldots i_{q}}:=U_{i_{0}} \cap U_{i_{1}} \cap \cdots \cap U_{i_{q}}$, which is a Stein open set.

With the conditions above, it is easy to check the following two claims.

Claim 4.2 ([9, Remark 2.19]). - For any E-valued $(n, q)$ form $u$ on $Y$ with measurable coefficients, $|u|_{\omega^{\prime}, h}^{2} \mathrm{~d} V_{\omega^{\prime}} \leqslant|u|_{\omega, h}^{2} \mathrm{~d} V_{\omega}$ holds. If $q=0$, $|u|_{\omega^{\prime}, h}^{2} \mathrm{~d} V_{\omega^{\prime}}=|u|_{\omega, h}^{2} \mathrm{~d} V_{\omega}$ holds.

Claim 4.3. - For any $q \geqslant 1$ and any $g \in L_{n, q}^{2}\left(U_{i_{0} i_{1} \ldots i_{q}} \backslash Z, E\right)_{\omega^{\prime}, h}$ such that $\bar{\partial} g=0$, there exists $f \in L_{n, q-1}^{2}\left(U_{i_{0} i_{1} \ldots i_{q}} \backslash Z, E\right)_{\omega^{\prime}, h}$ such that $\bar{\partial} f=g$ and

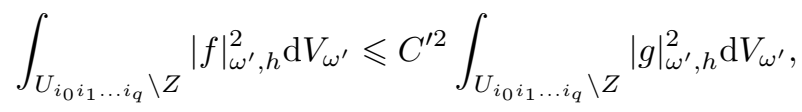

where $C^{\prime}:=\max _{i \in \Lambda} C_{i}$.

Since $U_{i_{0} i_{1} \ldots i_{q}} \backslash Z$ is a complete Kähler manifold and $\sqrt{-1} \Theta_{E, h}+$ $\sqrt{-1} \partial \bar{\partial} \varphi_{i_{0}} \geqslant \omega^{\prime} \otimes \operatorname{Id}_{E}$ holds on $U_{i_{0} i_{1} \ldots i_{q}} \backslash Z$, we can prove Claim 4.3 by Theorem 2.4.

Step 2: Construction of a homomorphism from Čech cohomology to Dolbeault cohomology. - We fix $c=\left\{c_{i_{0} i_{1} \ldots i_{q}}\right\} \in H^{q}\left(\mathcal{U}, K_{X} \otimes E(h)\right)$. By the definition of Čech cohomology, we have

(1) $c_{i_{0} i_{1} \ldots i_{q}} \in H^{0}\left(U_{i_{0} i_{1} \ldots i_{q}}, K_{X} \otimes E(h)\right)$ and

(2) $\delta c:=\left.\sum_{k=0}^{q+1}(-1)^{k} c_{i_{0} i_{1} \ldots \tilde{i}_{k} \ldots i_{q+1}}\right|_{U_{i_{0} i_{1} \ldots i_{q+1}}}=0$.

Let $\left\{\rho_{i}\right\}_{i \in \Lambda}$ be a partition of unity subordinate to $\mathcal{U}$. For each $k \in$ $\{0,1, \ldots, q-1\}$, we define an $E$-valued form $b_{i_{0} i_{1} \ldots i_{k}}$ by

$$
b_{i_{0} i_{1} \ldots i_{k}}:= \begin{cases}\sum_{j \in \Lambda} \rho_{j} c_{j i_{0} i_{1} \ldots i_{q-1}} & \text { if } k=q-1 \\ \sum_{j \in \Lambda} \rho_{j} \bar{\partial} b_{j i_{0} i_{1} \ldots i_{k}} & \text { otherwise. }\end{cases}
$$

Then, we have

$$
\begin{aligned}
\delta\left\{b_{i_{0} i_{1} \ldots i_{q-1}}\right\}_{i_{0} i_{1} \ldots i_{q}}=\sum_{k=0}^{q}(-1)^{k} b_{i_{0} i_{1} \ldots \check{i_{k}} \ldots i_{q}} \\
=\sum_{k=0}^{q} \sum_{j \in \Lambda}(-1)^{k} \rho_{j} c_{j i_{0} i_{1} \ldots \check{i_{k}} \ldots i_{q}}=\sum_{j \in \Lambda} \rho_{j} \sum_{k=0}^{q}(-1)^{k} c_{j i_{0} i_{1} \ldots \check{i_{k}} \ldots i_{q}}
\end{aligned}
$$

From $\delta c=0$, we have

$$
\sum_{j \in \Lambda} \rho_{j} \sum_{k=0}^{q}(-1)^{k} c_{j i_{0} i_{1} \ldots i_{k} \ldots i_{q}}=\sum_{j \in \Lambda} \rho_{j} c_{i_{0} i_{1} \ldots i_{q}}=c_{i_{0} i_{1} \ldots i_{q}} .
$$


Therefore, we obtain $\delta\left\{b_{i_{0} i_{1} \ldots i_{q-1}}\right\}=c$. Similarly we obtain $\delta\left\{b_{i_{0} i_{1} \ldots i_{k}}\right\}=$ $\left\{\bar{\partial} b_{i_{0} i_{1} \ldots i_{k+1}}\right\}$ for each $k \in\{0,1, \ldots, q-2\}$.

Therefore we obtain $\left.\bar{\partial} b_{i_{0}}\right|_{U_{i_{0}} \backslash Z}$, which is an $E$-valued $(n, q) \bar{\partial}$-closed form on $U_{i_{0}} \backslash Z$. Since we have

$$
\delta\left\{\bar{\partial} b_{i_{0}}\right\}=0 \quad \text { and } \quad \int_{U_{i_{0}} \backslash Z}\left|\bar{\partial} b_{i_{0}}\right|_{\omega^{\prime}, h}^{2} \mathrm{~d} V_{\omega^{\prime}} \leqslant \int_{U_{i_{0}}}\left|\bar{\partial} b_{i_{0}}\right|_{\omega, h}^{2} \mathrm{~d} V_{\omega}<+\infty
$$

by Claim 4.2, we can define $\alpha(c):=\left\{\bar{\partial} b_{i_{0}}\right\} \in L_{n, q}^{2}(Y, E)_{\omega^{\prime}, h} \cap \operatorname{Ker} \bar{\partial}$. By the above construction, we obtain the homomorphism

$$
\alpha: H^{q}\left(\mathcal{U}, K_{X} \otimes E(h)\right) \longrightarrow \frac{L_{n, q}^{2}(Y, E)_{\omega^{\prime}, h} \cap \operatorname{Ker} \bar{\partial}}{\operatorname{Im} \bar{\partial}} .
$$

Step 3: Construction of a homomorphism from Dolbeault cohomology to Čech cohomology. - We fix $u \in L_{n, q}^{2}(Y, E)_{\omega^{\prime}, h} \cap \operatorname{Ker} \bar{\partial}$ and define $D:=$ $\int_{Y}|u|_{\omega^{\prime}, h}^{2} \mathrm{~d} V_{\omega^{\prime}}<+\infty$. By Claim 4.3, there exists $v_{i_{0}} \in L_{n, q-1}^{2}\left(U_{i_{0}} \backslash Z, E\right)_{\omega^{\prime}, h}$ such that

$$
\bar{\partial} v_{i_{0}}=\left.u\right|_{U_{i_{0}} \backslash Z} \quad \text { and } \quad \int_{U_{i_{0}} \backslash Z}\left|v_{i_{0}}\right|_{\omega^{\prime}, h}^{2} \mathrm{~d} V_{\omega^{\prime}} \leqslant C^{2} D .
$$

We set $u^{1}:=\delta\left\{v_{i_{0}}\right\}$. From $\bar{\partial} u^{1}=0$, there exists $v_{i_{0} i_{1}} \in L_{n, q-2}^{2}\left(U_{i_{0} i_{1}} \backslash Z, E\right)_{\omega^{\prime}, h}$ such that

$$
\bar{\partial} v_{i_{0} i_{1}}=u_{i_{0} i_{1}}^{1} \quad \text { and } \quad \int_{U_{i_{0} i_{1} \backslash Z} \backslash}\left|v_{i_{0} i_{1}}\right|_{\omega^{\prime}, h}^{2} \mathrm{~d} V_{\omega^{\prime}} \leqslant 2 C^{\prime 2} D
$$

by Claim 4.3. We set $u^{2}:=\delta\left\{v_{i_{0} i_{1}}\right\}$ and we have $\bar{\partial} u^{2}=0$.

By repeating this procedure, we obtain $v_{i_{0} i_{1} \ldots i_{q-1}} \in L_{n, 0}^{2}\left(U_{i_{0} i_{1} \ldots i_{q-1}} \backslash Z\right.$, $E)_{\omega^{\prime}, h}$ and $u^{q}=\delta\left\{v_{i_{0} i_{1} \ldots i_{q-1}}\right\}$. By $\bar{\partial} u_{i_{0} i_{1} \ldots i_{q}}^{q}=0, u_{i_{0} i_{1} \ldots i_{q}}^{q}$ is a holomorphic $E$-valued $(n, 0)$ form on $U_{i_{0} i_{1} \ldots i_{q}} \backslash Z$. Since we obtain

$\int_{U_{i_{0} i_{1} \ldots i_{q}} \backslash Z}\left|u_{i_{0} i_{1} \ldots i_{q}}^{q}\right|_{\omega, h}^{2} \mathrm{~d} V_{\omega}=\int_{U_{i_{0} i_{1} \ldots i_{q}} \backslash Z}\left|u_{i_{0} i_{1} \ldots i_{q}}^{q}\right|_{\omega^{\prime}, h}^{2} \mathrm{~d} V_{\omega^{\prime}} \leqslant q ! C^{\prime 2} D<+\infty$ by Claim 4.2, $\left.u_{i_{0} i_{1} \ldots i_{q}}^{q}\right|_{U_{i_{0} i_{1} \ldots i_{q}} \backslash Z}$ extends on $U_{i_{0} i_{1} \ldots i_{q}}$ and $\left.u_{i_{0} i_{1} \ldots i_{q}}^{q}\right|_{U_{i_{0} i_{1} \ldots i_{q}} \backslash Z}$ is a holomorphic $E$-valued $(n, 0)$ form on $U_{i_{0} i_{1} \ldots i_{q}}$ by the Riemann extension theorem and Lemma 2.3. Therefore we can define

$$
\beta(u):=\left\{\left.u_{i_{0} i_{1} \ldots i_{q}}^{q}\right|_{U_{i_{0} i_{1} \ldots i_{q}} \backslash Z}\right\} \in H^{q}\left(\mathcal{U}, K_{X} \otimes E(h)\right) .
$$

By the above construction, we obtain the homomorphism

$$
\beta: \frac{L_{n, q}^{2}(Y, E)_{\omega^{\prime}, h} \cap \operatorname{Ker} \bar{\partial}}{\operatorname{Im} \bar{\partial}} \longrightarrow H^{q}\left(\mathcal{U}, K_{X} \otimes E(h)\right) .
$$

It is easy to check whether $\alpha$ and $\beta$ induce the isomorphism in Theorem 4.1. 
We finish this section by proving Theorem 1.3 and 1.4 .

Proof of Theorem 1.3. - By Theorem 4.1, we have $H^{q}\left(X, K_{X} \otimes E(h)\right) \cong$ $\frac{L_{n, q}^{2}(Y, E)_{\omega^{\prime}, h} \cap \operatorname{Ker} \bar{\partial}}{\operatorname{Im} \bar{\partial}}$. By Theorem 2.4, we have $\frac{L_{n, q}^{2}(Y, E)_{\omega^{\prime}, h} \cap \operatorname{Ker} \bar{\partial}}{\operatorname{Im} \bar{\partial}}=0$, which completes the proof.

Proof of Theorem 1.4. - By Theorem 1.2, $K_{X} \otimes E(h)$ is a coherent sheaf on $X$. Therefore, by the argument of [9, Claim 1], Theorem 2.5 and Theorem 4.1, we obtain $\overline{\operatorname{Im} \bar{\partial}}=\operatorname{Im} \bar{\partial}, \overline{\operatorname{Im} \bar{\partial}_{\omega^{\prime}, h}^{*}}=\operatorname{Im} \bar{\partial}_{\omega^{\prime}, h}^{*}$ and $H^{q}\left(X, K_{X} \otimes\right.$ $E(h)) \cong \mathcal{H}^{n, q}(Y, E)$. Similarly, we obtain $H^{q}\left(X, K_{X} \otimes L \otimes E(h)\right) \cong \mathcal{H}^{n, q}(Y$, $L \otimes E)$. By [9, Claim 2], the multiplication homomorphism $\times s: \mathcal{H}^{n, q}(Y, E) \rightarrow$ $\mathcal{H}^{n, q}(Y, L \otimes E)$ is well-defined and injective, which completes the proof.

\section{Applications}

\subsection{Hosono's example}

In this subsection, we study a singular Hermitian metric induced by holomorphic sections, proposed by Hosono [15, Chapter 4].

In this section, we assume that $E$ has holomorphic sections $s_{1}, \ldots, s_{N} \in$ $H^{0}(X, E)$ such that $E_{y}$ is generated by $s_{1}(y), \ldots, s_{N}(y)$ for a general point $y$. For any point $x \in X$, we take a local coordinate $\left(U ; z_{1}, \ldots, z_{n}\right)$ near $x$ and take a local holomorphic frame $e_{1}, \ldots, e_{r}$ of $E$ on $U$. Write $s_{i}=$ $\sum_{1 \leqslant j \leqslant r} f_{i j} e_{j}$, where $f_{i j}$ are holomorphic functions on $U$. A singular Hermitian metric $h$ induced by $s_{1}, \ldots, s_{N}$ is given by

$$
h_{j k}^{-1}:=\sum_{1 \leqslant i \leqslant N} \overline{f_{i j}} f_{i k} .
$$

By [15, Example 3.6 and Proposition 4.1], $h$ is positively curved and $E(h)$ is a coherent sheaf. Hosono pointed out that we can easily calculate the curvature of $h$ in the case $N=r$.

LEMMA 5.1. - In the case $N=r$, there exists a proper analytic subset $Z$ such that $\sqrt{-1} \Theta_{E, h}=0$ on $X \backslash Z$. In particular we obtain $\sqrt{-1} \Theta_{E, h} \geqslant 0$ on $X \backslash Z$ in the sense of Nakano.

Proof. - We take a finite Stein open covering $\left\{U_{i}\right\}_{i \in \Lambda}$. Under the conditions stated above, an $r \times r$ matrix $A^{(i)}$ on $U_{i}$ is defined by

$$
A_{j k}^{(i)}=f_{j k} .
$$

Set $Z_{i}:=\left\{z \in U_{i}: \operatorname{rank} A^{(i)}(z)<r\right\}$ and $W=\{z \in X: h$ is not smooth at $z\}$. We have $h=\left(h^{-1}\right)^{-1}=\frac{\widetilde{h^{-1}}}{\operatorname{det} h^{-1}}$, where $\widetilde{h^{-1}}$ is a cofactor matrix of $h^{-1}$. Since 
the $(i, j)$ element of $\widetilde{h^{-1}}$ is a smooth function on $X$ for any $1 \leqslant i, j \leqslant r$, we have $W=\left\{z \in X: \operatorname{det} h^{-1}(z)=0\right\}$. By [15, Lemma 4.3], we have

$$
\operatorname{det} h^{-1}=\sum_{1 \leqslant i_{1}<i_{2}<\cdots<i_{r} \leqslant N}\left|\operatorname{det}\left(s_{i_{1}}, s_{i_{2}}, \ldots, s_{i_{r}}\right)\right|,
$$

and therefore $W$ is a proper analytic subset. We write $Z:=\cup_{i \in \Lambda} Z_{i} \cup W$, which is a proper analytic subset.

By an easy computation, we have

$$
\sqrt{-1} \Theta_{E, h}=\sqrt{-1} \bar{\partial}\left(\bar{h}^{-1} \partial \bar{h}\right)=\sqrt{-1}\left(\partial \overline{\partial h}^{-1}-\partial \bar{h}^{-1} \bar{h}_{\bar{\partial}}^{-1}\right) \bar{h} .
$$

For any $z \in X \backslash Z$, we may assume $f_{i j}(z)=\delta_{i j}$. From $\bar{h}_{j k}^{-1}=\sum_{1 \leqslant i \leqslant r} f_{i j} \overline{f_{i k}}$, we have

$$
\partial \bar{h}_{j k}^{-1}(z)=\partial f_{k j}(z) \text { and } \quad \overline{\partial h}_{j k}^{-1}(z)=\overline{\partial f_{j k}}(z) .
$$

Thus, we obtain

$$
\left(\partial \bar{h}^{-1} \bar{h} \overline{\partial h}^{-1}\right)_{j k}(z)=\sum_{1 \leqslant i \leqslant r} \partial f_{i j} \overline{\partial f_{i k}}(z)=\partial \overline{\partial h}_{j k}^{-1}(z),
$$

which completes the proof.

By Lemma 5.1 and Theorem 1.3, we obtain the following corollary.

COROllary 5.2. - Let $\left(L, h_{L}\right)$ be a holomorphic line bundle with a singular Hermitian metric. We assume there exist a proper analytic subset $Z$ and a positive number $\epsilon>0$ such that $h_{L}$ is smooth on $X \backslash Z$ and $\sqrt{-1} \Theta_{L, h_{L}} \geqslant \epsilon \omega$ on $X$.

Then, $H^{q}\left(X, K_{X} \otimes L \otimes E\left(h h_{L}\right)\right)=0$ holds for all $q \geqslant 1$ for any holomorphic vector bundle $E$ and a singular Hermitian metric $h$ induced by $s_{1} \cdots s_{r} \in H^{0}(X, E)$.

In particular $H^{q}\left(X, K_{X} \otimes L \otimes E(h)\right)=0$ holds for all $q \geqslant 1$ if $L$ is ample.

We point out that such a metric $h_{L}$ on $L$ as in Corollary 5.2 always exists if $L$ is big.

Now, we introduce Hosono's example [15, Example 4.4]. Set $X=\mathbb{C}^{2}$ and let $E=X \times \mathbb{C}^{2}$ be the trivial rank-two bundle. We choose sections $s_{1}=e_{1}$ and $s_{2}=z e_{1}+w e_{2}$. Then the singular Hermitian metric $h_{E}$ induced by $s_{1}, s_{2}$ can be written by

$$
h_{E}=\frac{1}{|w|^{2}}\left(\begin{array}{cc}
|w|^{2} & -w \bar{z} \\
-z \bar{w} & |z|^{2}+1
\end{array}\right) .
$$

Hosono proved the following theorem by calculating the standard approximation by convolution of $h_{E}$. 
THEOREM 5.3 ([15, Theorem 1.2]). - The standard approximation defined by convolution of $h_{E}$ does not have a uniformly bounded curvature from below in the sense of Nakano.

Therefore, we can not apply the vanishing theorem of [5] to this example. However, we can apply Corollary 5.2 to this example. Thus our results are new results.

Remark 5.4. - We ask whether there exists a proper analytic subset $Z$ such that $\sqrt{-1} \Theta_{E, h} \geqslant 0$ on $X \backslash Z$ in the sense of Nakano for any singular Hermitian metric $h$ induced by $s_{1} \cdots s_{N} \in H^{0}(X, E)$ in the case $N>r$. This calculation is very complicated and this question is open, but it is likely that the answer is "No".

\subsection{Big vector bundles}

We review some of the standard facts on big vector bundles.

DEFinition 5.5 ([1, Section 2]). - Let $X$ be a smooth projective variety and $E$ be a holomorphic vector bundle. The base locus of $E$ is defined by

$$
\operatorname{Bs}(E):=\left\{x \in X: H^{0}(X, E) \rightarrow E_{x} \text { is not surjective }\right\},
$$

and the stable base locus of $E$ is defined by

$$
\mathbb{B}(E):=\bigcap_{m>0} \operatorname{Bs}\left(\operatorname{Sym}^{m} E\right),
$$

where $\operatorname{Sym}^{m}(E)$ is the $m$-th symmetric power of $E$. E by

Let $A$ be an ample line bundle. We define the augmented base locus of

$$
\mathbb{B}_{+}(E)=\bigcap_{p / q \in \mathbb{Q}>0} \mathbb{B}\left(\operatorname{Sym}^{q} E \otimes A^{-p}\right),
$$

where $\mathbb{Q}_{>0}$ is a set of positive rational numbers.

We point out $\mathbb{B}_{+}(E)$ do not depend on the choice of the ample line bundle $A$ by [1, Remark 2.7].

Definition 5.6 ([1, Theorem 1.1 and Section 6]).

(1) A vector bundle $E$ is said to be L-big if the tautological bundle $\mathcal{O}_{\mathbb{P}(E)}(1)$ on $\mathbb{P}(E)$ is big.

(2) A vector bundle $E$ is said to be $\mathrm{V}$-big if $\mathbb{B}_{+}(E) \neq X$. 
We note that if $E$ is V-big then it is L-big as well by [1, Corollary 6.5]. We will denote by $\pi: \mathbb{P}(E) \rightarrow X$ the canonical projection and by $\widetilde{\omega}$ a Kähler form on $\mathbb{P}(E)$. Inayama communicated to the author the following lemma.

LEMMA 5.7. - Let $E$ be an L-big vector bundle and $\widetilde{h}$ be a singular Hermitian metric on $\mathcal{O}_{\mathbb{P}(E)}(1)$. We assume that there exist a positive number $\epsilon>0$ and a proper analytic subset $\widetilde{Z} \subset \mathbb{P}(E)$ such that $\widetilde{h}$ is smooth on $\mathbb{P}(E) \backslash \widetilde{Z}, \pi(\widetilde{Z}) \neq X$, and $\sqrt{-1} \Theta_{\mathcal{O}_{\mathbb{P}(E)}(1), \tilde{h}} \geqslant \epsilon \widetilde{\omega} \otimes \operatorname{Id}_{\mathcal{O}_{\mathbb{P}(E)}(1)}$.

Then $\widetilde{h}$ induces a singular Hermitian metric $h_{m}$ on $\operatorname{Sym}^{m}(E) \otimes \operatorname{det} E$ such that

(1) $h_{m}$ is smooth on $X \backslash \pi(\widetilde{Z})$,

(2) $h_{m}$ is a positively curved singular Hermitian metric, and

(3) $\sqrt{-1} \Theta_{\operatorname{Sym}^{m}(E) \otimes \operatorname{det} E, h_{m}} \geqslant \epsilon \omega \otimes \operatorname{Id}_{\operatorname{Sym}^{m}(E) \otimes \operatorname{det} E}$ on $X \backslash \pi(\widetilde{Z})$ in the sense of Nakano.

Proof. - From $\operatorname{Sym}^{m}(E) \otimes \operatorname{det} E=\pi_{*}\left(K_{\mathbb{P}(E) / X} \otimes \mathcal{O}_{\mathbb{P}(E)}(m+r)\right)$, $\operatorname{Sym}^{m}(E) \otimes \operatorname{det} E$ can be endowed with the $L^{2}$ metric $h_{m}$ with respect to $\widetilde{h}$. Therefore by the argument of [2, Theorem 1.2, Theorem 1.3, and Section 4], (1) and (3) are proved. By [14] and [19], (2) is proved.

Remark 5.8. - By [1, Proposition 3.2], $\pi\left(\mathbb{B}_{+}\left(\mathcal{O}_{\mathbb{P}(E)}(1)\right)\right)=\mathbb{B}_{+}(E)$ holds. Therefore if $E$ is V-big, such a metric $\widetilde{h}$ on $\mathcal{O}_{\mathbb{P}(E)}(1)$ as in the assumption of Lemma 5.7 always exists.

Thus, we can apply Theorem 1.3 to $\left(\operatorname{Sym}^{m}(E) \otimes \operatorname{det} E, h_{m}\right)$ and we have the following corollary.

Corollary 5.9. - Under the conditions stated in Lemma 5.7, $H^{q}(X$, $\left.K_{X} \otimes\left(\operatorname{Sym}^{m}(E) \otimes \operatorname{det} E\right)\left(h_{m}\right)\right)=0$ holds for any $m \geqslant 0$ and $q \geqslant 1$.

This corollary is a generalization of Griffiths' vanishing theorem in [13].

\section{On Ohsawa's vanishing theorem}

We use the results of [17]. Let $Y$ be a complete Kähler manifold, $\omega^{\prime}$ be a Kähler form and $(E, h)$ be a vector bundle with a smooth Hermitian metric. Let $\tau$ be a smooth semipositive $(1,1)$ form on $Y$. Write

$$
L_{n, q}^{2}(Y, E)_{\tau, h}:=\left\{f \in L_{n, q}^{2}(Y, E)_{\omega^{\prime}+\tau, h} ; \lim _{\epsilon \downarrow 0} \int_{Y}|f|^{2}{ }_{\epsilon \omega^{\prime}+\tau, h} \mathrm{~d} V_{\epsilon \omega^{\prime}+\tau}<+\infty\right\} .
$$

By [17, Proposition 2.4], $\lim _{\epsilon \downarrow 0} \int_{Y}|f|_{\epsilon \omega^{\prime}+\tau, h}^{2} \mathrm{~d} V_{\epsilon \omega^{\prime}+\tau}$ and $L_{n, q}^{2}(Y, E)_{\tau, h}$ do not depend on the choice of the metric $\omega^{\prime}$. We use Ohsawa's $L^{2}$ estimate. 
THEOREM 6.1 ([17, Theorem 2.8]). - Under the conditions stated above, we also assume that $\sqrt{-1} \Theta_{E, h}-\tau \otimes \operatorname{Id}_{E} \geqslant 0$ on $Y$. For any $q \geqslant 1$ and $f \in L_{n, q}^{2}(Y, E)_{\tau, h}$ such that $\bar{\partial} f=0$, there exists $g \in L_{n, q-1}^{2}(Y, E)_{\tau, h}$ such that $\bar{\partial} g=f$ and

$$
\lim _{\epsilon \downarrow 0} \int_{Y}|g|_{\epsilon \omega^{\prime}+\tau, h}^{2} \mathrm{~d} V_{\epsilon \omega^{\prime}+\tau} \leqslant q \lim _{\epsilon \downarrow 0} \int_{Y}|f|_{\epsilon \omega^{\prime}+\tau, h}^{2} \mathrm{~d} V_{\epsilon \omega^{\prime}+\tau} .
$$

Now we prove Theorem 1.5.

Proof. - We take a complete Kähler form $\omega^{\prime}$ on $Y:=X \backslash Z$ as in Section 4. The proof of Theorem 1.5 is similar to those of [17, Theorem 3.1] and Theorem 4.1 with a slight modification.

Let $\mathcal{U}=\left\{U_{j}\right\}_{j \in \Lambda}$ be a finite Stein cover of $W$. By Theorem 1.2 and the Grauert direct image theorem, $\pi_{*}\left(K_{X} \otimes E(h)\right)$ is coherent. Therefore the sheaf cohomology $H^{q}\left(W, \pi_{*}\left(K_{X} \otimes E(h)\right)\right)$ is isomorphic to the Cech cohomology $H^{q}\left(\mathcal{U}, \pi_{*}\left(K_{X} \otimes E(h)\right)\right)$. We point out the following claim.

Claim $6.2\left(\left[17\right.\right.$, Lemma 3.2]). - For any form $g$ on $W,\left|\pi^{*} g(x)\right|_{\omega+\pi^{*} \sigma} \leqslant$ $|g(\pi(x))|_{\sigma}$ holds at any $x \in X$.

We fix $c=\left\{c_{i_{0} i_{1} \ldots i_{q}}\right\} \in H^{q}\left(\mathcal{U}, \pi_{*}\left(K_{X} \otimes E(h)\right)\right)$. By the definition of Čech cohomology, we have

(1) $c_{i_{0} i_{1} \ldots i_{q}} \in H^{0}\left(U_{i_{0} i_{1} \ldots i_{q}}, \pi_{*}\left(K_{X} \otimes E(h)\right)\right)=H^{0}\left(\pi^{-1}\left(U_{i_{0} i_{1} \ldots i_{q}}\right), K_{X} \otimes\right.$ $E(h))$ and

(2) $\delta c:=\left.\sum_{k=0}^{q+1}(-1)^{k} c_{i_{0} i_{1} \ldots \check{i_{k}} \ldots i_{q+1}}\right|_{\pi^{-1}\left(U_{i_{0} i_{1} \ldots i_{q+1}}\right)}=0$.

Let $\left\{\rho_{j}\right\}_{j \in \Lambda}$ be a partition of unity of $\mathcal{U}$. Based on Section 4, for each $k \in\{0,1, \ldots, q-1\}$, we define an $E$-valued form $b_{i_{0} i_{1} \ldots i_{k}}$ by

$$
b_{i_{0} i_{1} \ldots i_{k}}:= \begin{cases}\sum_{j \in \Lambda} \pi^{*}\left(\rho_{j}\right) c_{j i_{0} i_{1} \ldots i_{q-1}} & \text { if } k=q-1 \\ \sum_{j \in \Lambda} \pi^{*}\left(\rho_{j}\right) \bar{\partial} b_{j i_{0} i_{1} \ldots i_{k}} & \text { otherwise. }\end{cases}
$$

As in Step 2 in the proof of Theorem 4.1, we obtain

$$
\delta\left\{b_{i_{0} i_{1} \ldots i_{q-1}}\right\}=c, \quad \text { and } \quad \delta\left\{b_{i_{0} i_{1} \ldots i_{k}}\right\}=\left\{\bar{\partial} b_{i_{0} i_{1} \ldots i_{k+1}}\right\}
$$

for each $k \in\{0,1, \ldots, q-2\}$.

Therefore we obtain $\left.\bar{\partial} b_{i_{0}}\right|_{\pi^{-1}\left(U_{i_{0}}\right) \backslash Z}$, which is an $E$-valued $(n, q) \bar{\partial}$-closed form on $\pi^{-1}\left(U_{i_{0}}\right) \backslash Z$. By Claim $6.2,\left|\bar{\partial}\left(\pi^{*} \rho_{j}\right)\right|_{\epsilon \omega+\pi^{*} \sigma}$ are bounded above by $\left|\bar{\partial}\left(\rho_{j}\right)\right|_{\sigma}$ for any $\epsilon>0$ and $\left|c_{i_{0} i_{1} \ldots i_{q}}\right|_{\epsilon \omega+\pi^{*} \sigma}^{2} \mathrm{~d} V_{\epsilon \omega+\pi^{*} \sigma}$ are independent of $\epsilon$ by 
Claim 4.2. Therefore we have $\delta\left\{\bar{\partial} b_{i_{0}}\right\}=0$ and

$$
\begin{aligned}
\int_{\pi^{-1}\left(U_{i_{0}}\right) \backslash Z}\left|\bar{\partial} b_{i_{0}}\right|_{\epsilon \omega^{\prime}+\pi^{*} \sigma, h}^{2} \mathrm{~d} V_{\epsilon \omega^{\prime}+\pi^{*} \sigma} & \\
& \leqslant \int_{\pi^{-1}\left(U_{i_{0}}\right)}\left|\bar{\partial} b_{i_{0}}\right|_{\epsilon \omega+\pi^{*} \sigma, h}^{2} \mathrm{~d} V_{\epsilon \omega+\pi^{*} \sigma} \\
& \leqslant \lim _{\epsilon \downarrow 0} \int_{\pi^{-1}\left(U_{i_{0}}\right)}\left|\bar{\partial} b_{i_{0}}\right|_{\epsilon \omega+\pi^{*} \sigma, h}^{2} \mathrm{~d} V_{\epsilon \omega+\pi^{*} \sigma} \\
& <+\infty
\end{aligned}
$$

for any $\epsilon>0$ by Claim 4.2. Thus, we may regard $\left\{\bar{\partial} b_{i_{0}}\right\}$ as an element of $L_{n, q}^{2}(Y, E)_{\sigma, h}$ and denote by $b:=\bar{\partial} b_{i_{0}}$. By Theorem 6.1 , there exists $a \in L_{n, q-1}^{2}(Y, E)_{\sigma, h}$ such that

$$
\bar{\partial} a=b \quad \text { and } \quad \lim _{\epsilon \downarrow 0} \int_{Y \backslash Z}|a|_{\epsilon \omega^{\prime}+\pi^{*} \sigma, h}^{2} \mathrm{~d} V_{\epsilon \omega^{\prime}+\pi^{*} \sigma}<+\infty .
$$

Write $d_{i_{0}}^{1}:=b_{i_{0}}-a \in L_{n, q-1}^{2}\left(\pi^{-1}\left(U_{i_{0}}\right) \backslash Z, E\right)_{\sigma, h}$ and $d^{1}:=\left\{d_{i_{0}}^{1}\right\}$. We point out

$$
\delta d^{1}=\delta\left\{b_{i_{0}}\right\}=\left\{\bar{\partial} b_{i_{0} i_{1}}\right\} \quad \text { and } \quad \bar{\partial} d^{1}=0 .
$$

By Theorem 6.1, there exists $a_{i_{0}} \in L_{n, q-2}^{2}\left(\pi^{-1}\left(U_{i_{0}}\right) \backslash Z, E\right)_{\sigma, h}$ such that

$$
\bar{\partial} a_{i_{0}}=d_{i_{0}}^{1} \quad \text { and } \quad \lim _{\epsilon \downarrow 0} \int_{U_{i_{0}} \backslash Z}\left|a_{i}\right|_{\epsilon \omega^{\prime}+\pi^{*} \sigma, h}^{2} \mathrm{~d} V_{\epsilon \omega^{\prime}+\pi^{*} \sigma}<+\infty \text {. }
$$

We write $d_{i_{0} i_{1}}^{2}:=b_{i_{0} i_{1}}-a_{i_{0}}+a_{i_{1}} \in L_{n, q-2}^{2}\left(\pi^{-1}\left(U_{i_{0} i_{1}}\right) \backslash Z, E\right)_{\sigma, h}$ and $d^{2}:=$ $\left\{d_{i_{0} i_{1}}^{2}\right\}$. We point out that

$$
\delta d^{2}=\delta\left\{b_{i_{0} i_{1}}\right\}=\left\{\bar{\partial} b_{i_{0} i_{1} i_{2}}\right\} \quad \text { and } \quad \bar{\partial} d^{2}=0 .
$$

By repeating this procedure, we obtain $d_{i_{0} i_{1} \ldots i_{q-1}}^{q-1} \in L_{n, 0}^{2}\left(\pi^{-1}\left(U_{i_{0} i_{1} \ldots i_{q-1}}\right) \backslash Z\right.$, $E)_{\sigma, h}$ and $d^{q-1}:=\left\{d_{i_{0} i_{1} \ldots i_{q-1}}^{q-1}\right\}$ such that

$$
\delta d^{q-1}=\delta\left\{b_{i_{0} i_{1} \ldots i_{q-1}}\right\}=c \text { and } \bar{\partial} d^{q-1}=0 .
$$

We have

$$
\begin{aligned}
\int_{\pi^{-1}\left(U_{i_{0} i_{1} \ldots i_{q-1}}\right) \backslash Z} & \left|d_{i_{0} i_{1} \ldots i_{q-1}}^{q-1}\right|_{\omega, h}^{2} \mathrm{~d} V_{\omega} \\
& =\int_{\pi^{-1}\left(U_{i_{0} i_{1} \ldots i_{q-1}}\right) \backslash Z}\left|d_{i_{0} i_{1} \ldots i_{q-1}}^{q-1}\right|_{\omega^{\prime}+\pi^{*} \sigma, h}^{2} \mathrm{~d} V_{\omega^{\prime}+\pi^{*} \sigma} \\
& =\lim _{\epsilon \downarrow 0} \int_{\pi^{-1}\left(U_{i_{0} i_{1} \ldots i_{q-1}}\right) \backslash Z}\left|d_{i_{0} i_{1} \ldots i_{q-1}}^{q-1}\right|_{\epsilon \omega^{\prime}+\pi^{*} \sigma, h}^{2} \mathrm{~d} V_{\epsilon \omega^{\prime}+\pi^{*} \sigma} \\
& <+\infty .
\end{aligned}
$$


By Lemma 2.3 and the Riemann extension theorem, $d_{i_{0} i_{1} \ldots i_{q-1}}^{q-1}$ extends on $\pi^{-1}\left(U_{i_{0} i_{1} \ldots i_{q-1}}\right)$ and $d_{i_{0} i_{1} \ldots i_{q-1}}^{q-1}$ is holomorphic on $\pi^{-1}\left(U_{i_{0} i_{1} \ldots i_{q-1}}\right)$. Therefore we obtain $d_{i_{0} i_{1} \ldots i_{q-1}}^{q-1} \in H^{0}\left(\pi^{-1}\left(U_{i_{0} i_{1} \ldots i_{q-1}}\right), K_{X} \otimes E(h)\right)$ and $\delta d^{q-1}=c$, which completes the proof.

Remark 6.3. - We ask whether, under the assumptions of singular Hermitian metrics as in Theorems 1.3-1.5, we can show higher rank analogies of a generalization of the Kollár-Ohsawa type vanishing theorem by Matsumura [16], an injectivity theorem of higher direct images by Fujino [10], an injectivity theorem of pseudoeffective line bundles by Fujino and Matsumura [11] and so on. It is likely the answer is "Yes" and the proof may be similar to the original proof with a slight modification.

\section{Bibliography}

[1] T. Bauer, S. J. Kovács, A. Küronya, E. C. Mistretta, T. Szemberg \& S. Urbinati, "On positivity and base loci of vector bundles", Eur. J. Math. 1 (2015), no. 2, p. 229-249.

[2] B. Berndtsson, "Curvature of vector bundles associated to holomorphic fibrations", Ann. Math. 169 (2009), no. 2, p. 531-560.

[3] B. Berndtsson \& M. PĂUn, "Bergman kernels and the pseudoeffectivity of relative canonical bundles", Duke Math. J. 145 (2008), no. 2, p. 341-378.

[4] J. CAO \& M. PĂUN, "Kodaira dimension of algebraic fiber spaces over abelian varieties", Invent. Math. 207 (2017), no. 1, p. 345-387.

[5] M. A. A. de Cataldo, "Singular Hermitian metrics on vector bundles", J. Reine Angew. Math. 502 (1998), p. 93-122.

[6] J.-P. Demailly, "Complex analytic and differential geometry", https:// www-fourier.ujf-grenoble.fr/ demailly/manuscripts/agbook.pdf.

[7] — , "Estimations $L^{2}$ pour l'opérateur $\bar{\partial}$ d'un fibré vectoriel holomorphe semipositif au-dessus d'une variété kählérienne complète", Ann. Sci. Éc. Norm. Supér. 15 (1982), no. 3, p. 457-511.

[8] - Analytic methods in algebraic geometry, Surveys of Modern Mathematics, vol. 1, International Press; Higher Education Press, 2012, viii+231 pages.

[9] O. Fujino, "A transcendental approach to Kollár's injectivity theorem", Osaka J. Math. 49 (2012), no. 3, p. 833-852.

[10] - "A transcendental approach to Kollár's injectivity theorem II", J. Reine Angew. Math. 681 (2013), p. 149-174.

[11] O. Fujino \& S.-I. Matsumura, "Injectivity theorem for pseudo-effective line bundles and its applications", https://arxiv.org/abs/1605.02284, 2017.

[12] H. Grauert \& R. Remmert, Coherent analytic sheaves, Grundlehren der Mathematischen Wissenschaften, vol. 265, Springer, 1984, xviii+249 pages.

[13] P. A. Griffiths, "Hermitian differential geometry, Chern classes, and positive vector bundles", in Global Analysis (Papers in Honor of K. Kodaira), University of Tokyo Press, 1969, p. 185-251.

[14] C. Hacon, M. Popa \& C. Schnell, "Algebraic fiber spaces over abelian varieties: around a recent theorem by Cao and Puaun", in Local and global methods in algebraic geometry, Contemporary Mathematics, vol. 712, American Mathematical Society, 2018, p. 143-195. 
Nadel-Nakano vanishing theorems of vector bundles with singular Hermitian metrics

[15] G. Hosono, "Approximations and examples of singular Hermitian metrics on vector bundles", Ark. Mat. 55 (2017), no. 1, p. 131-153.

[16] S.-I. Matsumura, "A vanishing theorem of Kollár-Ohsawa type", Math. Ann. 366 (2016), no. 3-4, p. 1451-1465.

[17] T. Ohsawa, "Vanishing theorems on complete Kähler manifolds", Publ. Res. Inst. Math. Sci. 20 (1984), no. 1, p. 21-38.

[18] M. PĂUN, "Singular Hermitian metrics and positivity of direct images of pluricanonical bundles", in Algebraic geometry: Salt Lake City 2015, Proceedings of Symposia in Pure Mathematics, vol. 97, American Mathematical Society, 2018, p. 519-553.

[19] M. PăUn \& S. TAKayama, "Positivity of twisted relative pluricanonical bundles and their direct images", J. Algebr. Geom. 27 (2018), no. 2, p. 211-272.

[20] H. Raufi, "Singular hermitian metrics on holomorphic vector bundles", Ark. Mat. 53 (2015), no. 2, p. 359-382. 